\title{
Prevalence and associated risk factors of peripheral artery disease in virologically suppressed HIV-infected individuals on antiretroviral therapy in Kwara state, Nigeria: a cross sectional study
}

\author{
Chidozie Elochukwu Agu', Ikenna Kingsley Uchendu ${ }^{2 *}$, Augusta Chinyere Nsonwu',
}

Chukwugozie Nwachukwu Okwuosa ${ }^{2}$ and Peter Uwadiegwu Achukwu ${ }^{2}$

\begin{abstract}
Background: The association between HIV and cardiovascular disease (CVD) has been reported in several studies. However, there is paucity of information on the prevalence of subclinical disease as well as its associated risk factors in sub-Saharan African population. The aim of this study was to determine the prevalence and associated risk factors of peripheral artery disease (PAD) among virologically suppressed HIV-infected participants in Kwara State, Nigeria.

Methods: This study was conducted between July 2018 and December 2018. A total of 150 HIV-infected participants aged between 20 and 55 years and 50 HIV non-infected age-matched controls were randomly recruited in the study. Sociodemographic, anthropometric and clinical data were collected using a well-structured questionnaire. Ankle brachial index (ABI) was measured, PAD was defined as ABI of $<0.9$. Cryopreserved serum was used to evaluate lipid profile parameters. Student's t-test and Chi-square were used to compare continuous and categorical variables. Associations of CVD risk factors and clinical data, and lipid profile with low ABI were assessed using logistic regression analysis.

Results: The study participants had a mean age of $43.73 \pm 8.74$, majority were females (72.7\%) with a mean duration on ART of $7.73 \pm 3.52$ years. Hypertension was present in $15.9 \%$, diabetes $4 \%$, family history of CVD $8.6 \%$ and metabolic syndrome $17.3 \%$ in the study group. The study participants recorded significantly lower mean values for $\mathrm{ABI}, \mathrm{HDL}-\mathrm{C}$ and significantly higher mean values of $\mathrm{TG}(P<0.05)$ compared to the control group. The prevalence of low $\mathrm{ABI}(14.6 \%)$ was higher in the study group compared to the control group (2\%). A significantly negative correlation between $\mathrm{ABI}$ and duration on $\mathrm{ART}(r=-0.163, P=0.041)$ and a positive correlation between viral load and TG were observed in the study group. TC (OR 1.784, $P=0.011)$, LDL-C (OR 1.824, $P=0.010)$ and CD4 cell count $<200$ cells $/ \mathrm{mm}^{3}$ (OR 2.635, $P=0.364$ ) were associated with low ABI in the participants.
\end{abstract}

Conclusion: Viral suppression with combined antiretroviral therapy and long term treatment is associated with dyslipidaemia, with increased risk of PAD. Prevalence of PAD in virologically-suppressed individuals does not differ from the controls in the population studied.

Keywords: HIV, Antiretroviral therapy, Cardiovascular disease, Metabolic syndrome, Dyslipidemia

\footnotetext{
* Correspondence: Ikenna.uchendu@unn.edu.ng

${ }^{2}$ Department of Medical Laboratory Science, Faculty of Health Science and

Technology, College of Medicine, University of Nigeria Enugu Campus,

Enugu, Nigeria

Full list of author information is available at the end of the article
}

(c) The Author(s). 2019 Open Access This article is distributed under the terms of the Creative Commons Attribution 4.0 International License (http://creativecommons.org/licenses/by/4.0/), which permits unrestricted use, distribution, and

reproduction in any medium, provided you give appropriate credit to the original author(s) and the source, provide a link to the Creative Commons license, and indicate if changes were made. The Creative Commons Public Domain Dedication waiver (http://creativecommons.org/publicdomain/zero/1.0/) applies to the data made available in this article, unless otherwise stated. 


\section{Background}

In developed countries, there is significant improvement in the prognosis of People Living with HIV (PLHIV), who receive antiretroviral treatment (ART); as a result of the availability of well-established access to care and ART [1, 2]. In the last 20 years, there has been consistent decline in the incidence and mortality of AIDS-defining illnesses linked to severe end-stage immune suppression, whereas the role of non-AIDS comorbidities has risen [3-6]. These reports have come mainly from high- and middle-income countries $[7,8]$, with very few reports from low-income countries, particularly sub-Saharan Africa $[9,10]$. Although reports from sub-Saharan Africa indicated that tuberculosis, sepsis, advanced HIV disease and pulmonary infections are the leading causes of death [11]. Furthermore, CVD (cardiovascular disease) is now increasingly implicated as the cause of death among HIV-infected individuals in sub-Saharan Africa.

Greater prevalence and risk of CVD has been reported in long-term-treated patients than age-matched uninfected adults, without sufficient knowledge for the reason [7], and the underlying mechanism causing the high risk of non-AIDS complications is most likely multifactorial and includes comorbid conditions and toxicity from antiretroviral therapy $[12,13]$.

Although the relative risk of CVD is increased in patients with HIV, one limitation in studying CVD in this generally young patient population has been the relatively low absolute event rate in terms of CVD deaths [14]. Peripheral artery disease (PAD) is highly associated with atherosclerosis and is a predictor of cardiovascular outcomes [14-17]. Ankle brachial index (ABI) has been used to document the burden of subclinical CVD, and better understand the underlying pathophysiological mechanisms, and follow response to treatment interventions. Ankle-brachial index $(\mathrm{ABI})$ is the screening tool for PAD. An $A B I \leq 0.9$ (low $A B I$ ) is diagnostic of PAD [18]. The ABI between 0.91-0.99 (borderline values) is significantly considered prognostic in higher cardiovascular mortality. The $A B I \geq 1.3$ (high $A B I$ ) suggests rigidity and non-compressibility of lower limb arteries. Abnormal ABI value in asymptomatic patients is associated with a higher incidence of CVD [19].

This study was aimed to evaluate the prevalence and associated risk factors of peripheral artery disease (PAD) in virologically suppressed HIV-infected participants in Ilorin, Kwara State, Nigeria.

\section{Methods}

\section{Study area}

The study was conducted in Kwara State, a State in western Nigeria, located within the North central geopolitical zone, commonly referred to as the Middle belt with a population of 2,365,353 [20]. The primary ethnic group is Yoruba, with significant Nupe, Bariba and Fulani minorities. Kwara State consists of sixteen (16) Local Government Areas. They are: Asa, Baruten, Edu, Ekiti, Ifelodun, Ilorin East, Ilorin South, Ilorin West, Irepodun, Isin, Kaiama, Moro, Offa, OkeEro, Oyun and Pategi.

\section{Study design}

A cross sectional study design was adopted for this study. Informed consents were obtained from all participants before being enrolled into the study. Socio-demographic and behavioral data were collected using a well-structured questionnaire after which blood samples were drawn from the participants. The study partic.ipants include HIV-positive male and females aged between 20 and 50 years who were attending HIV clinic in University of Ilorin Teaching Hospital (UITH) and Sabo Oke medical center, Ilorin, Kwara state for routine checkup, and antiretroviral therapy (ART) pick up and control group of un-infected health participants resident within the same region. The study was conducted in six (6) months between July 2018 and December 2018.

\section{Sample size determination}

The sample size was calculated using Fischer's formula

Sample size $(\mathrm{n})=\frac{\mathrm{Z}^{2} \mathrm{P}(1-\mathrm{P})}{\mathrm{D}^{2}}$

Using a prevalence of $11 \%$ (prevalence of cardiovascular disease among people living with HIV from the Data Collection on Adverse Events of Anti-HIV Drugs (D:A:D study) [21].

$\mathrm{Z}=$ statistics for the level of $95 \%$ confidence interval (1.96)

$\mathrm{P}=$ prevalence

$\mathrm{D}=$ Desired degree of accuracy; here taken to be 0.05

Sample size $(\mathrm{n})=\frac{(1.96)^{2} \mathrm{P}(1-\mathrm{P})}{\mathrm{D}^{2}}$

$$
\begin{aligned}
& \mathrm{n}=\frac{3.84 \times 0.11(1-0.11)}{0.05 \times 0.05} \\
& \mathrm{n}=\frac{3.84 \times 0.11(0.89)}{0.0025} \\
& \mathrm{n}=150
\end{aligned}
$$

Thus, a total of $150 \mathrm{HIV}$ - infected participants who have been on antiretroviral therapy (ART) for 6 months and above were recruited into the study.

\section{Subject selection}

A total of 200 participants, made up of $150 \mathrm{HIV}$-infected participants and 50 un-infected control groups within the age range of $20-50$ years, were randomly recruited in the study. This study was conducted between July 2018 and December 2018. The study group participants were recruited from Sabo Oke Medical Center Ilorin; a Global Fund supported site and the ART treatment and 
care unit, University of Ilorin Teaching Hospital (UITH) supported by PEPFAR (President's Emergency Plan for AIDS Relief). Medical history along with physical examination, including height, weight, waist and hip measurement, brachial and ankle blood pressure were obtained for all the participants enrolled in the study.

\section{Inclusion criteria}

HIV-infected adults presently on stable ART for at least 6 months without a known coronary heart disease (CHD) or diabetes mellitus and with a viral RNA $<1000$ copies/ml, between the ages of $18-50$ years.

\section{Exclusion criteria}

HIV-infected adults who have defaulted from ART use, with a known coronary heart disease or diabetes mellitus, those who have been on statin for at least 6 months, those with known co-infections such as tuberculosis, hepatitis B and C, with a viral RNA $>1000$ copies/ml or those with advanced symptoms of the disease i.e. AIDS.

\section{Data collection tools}

The questionnaire used in this study was specifically developed for this study (Additional file 1). A detailed questionnaire interview was conducted to collect personal data on each participant enrolled in the study, detailing his/her demographic characteristics, family history of cardiovascular diseases was based on the National Cholesterol Education Program Adult Treatment Panel III (NCEP ATP III) criteria, past and current medication usage, presence of co-infections and HIV disease characteristics (duration of HIV infection, duration of ART treatment, viral RNA count, nadir and current CD4+ T-cell count, and past and current ART regimen) [22].

\section{Ankle brachial index $(\mathrm{ABI})$ measurement}

The ABI was determined using a hand-held continuous wave doppler device $8 \mathrm{MHz}$ probe, as described by Kwiatkowska et al. [22] and Olalla et al. [23]. Briefly, the examination was done after $5 \mathrm{~min}$ of rest in the supine position. A pressure cuff was used, placed on either arm and then above the ankles. The probe was placed at the cubital fossa, medial malleolus and dorsal foot for systolic blood pressure detection at the brachial artery, left posterior tibial and dorsalis pedis artery respectively. The cuff was inflated to $20 \mathrm{~mm}$ above the audible pulse signal and then slowly emptied until the first pulse signal was detected. Ankle brachial index value was determined by taking the higher pressure of the two arteries at the ankles divided by the highest brachial systolic blood pressures. Using the formula below, ABI was calculated as:

$$
\mathrm{ABI}=\frac{\text { Highest systolic blood pressure in the Ankles }}{\text { Highest systolic blood pressure in both arms }}
$$

Interpretation of ankle brachial index measurements: Normal ABI $=1.0-1.4$

Borderline ABI $=0.91-0.99$,

$\mathrm{PAD}=<0.9$

\section{Anthropometric measurements}

Anthropometric parameters such as height $(\mathrm{m})$, weight $(\mathrm{kg})$, waist circumference $(\mathrm{cm})$, hip circumference $(\mathrm{cm})$ were determined as described by Schienkiewitz et al. [24]. Briefly, weight and height were measured with the subjects wearing light clothing and without shoes.Weight was estimated in kilogram using a balanced scale; height was measured in meters using a wallmounted ruler with the participant standing with feet together and with head, shoulder, buttocks and heels touching the wall. Waist and hip circumference were estimated to the nearest $0.1 \mathrm{~cm}$ using a flexible but inelastic measuring tape; waist circumference was taken between the costal margin and the iliac crest in the mid-auxillary line around the gluteal region [24, 25]. Body mass index was calculated as weight in kilogram divided by the square of the height in metres $\left(\mathrm{kg} / \mathrm{m}^{2}\right)$, waist-to-hip (WHR) was calculated by dividing the measurement of the waist $(\mathrm{cm})$ by that of the hip $(\mathrm{cm})$ and was used together with waist circumference as an index of central obesity.

The following definitions were used, general obesity a BMI of $\geq 30 \mathrm{~kg} / \mathrm{m}^{2}$, central obesity - a waist circumference of $\geq 80 \mathrm{~cm}$ in women or $\geq 94 \mathrm{~cm}$ in men, waist-tohip ratio of $\geq 0.90$ in men or $\geq 0.85$ in women. Normal weight, as BMI of $18.5-24.9 \mathrm{~kg} / \mathrm{m}^{2}$ [25].

\section{Definition of cardiovascular disease (CVD) risk factors Dyslipidaemia}

Dyslipidaemia was defined as either elevated TG levels $\geq 1.7 \mathrm{mmol} / \mathrm{l}(150 \mathrm{mg} / \mathrm{dl})$, reduced HDL-cholesterol $<0.9$ $\mathrm{mmol} / \mathrm{l}(40 \mathrm{mg} / \mathrm{dl})$ formen and $<1.29 \mathrm{mmol} / \mathrm{l}(50 \mathrm{mg} / \mathrm{dl})$ in women, or specific treatment for previously identified hypertriglyceridaemia and/or reduced HDL-cholesterol (NCEP criteria).

\section{Elevated blood pressure/hypertension}

Elevated blood pressure was defined as systolic pressure $\geq 130 \mathrm{mmHg}$, diastolic $\geq 85 \mathrm{mmHg}$, or antihypertensive therapy for initially detected hypertension [26]. 


\section{Hyperglycaemia}

Hyperglycaemia was defined as the presence of either fasting plasma glucose of $(\geq 5.6 \mathrm{mmol} / \mathrm{l}$ or $100 \mathrm{mg} / \mathrm{dl})$ or previously detected diabetes [27].

\section{Metabolic syndrome (MetS)}

Participants defined as having the MetS, had central obesity (defined as waist circumference) $\geq 94 \mathrm{~cm}$ for males and $\geq 80 \mathrm{~cm}$ for females in addition with any two of four factors. These four factors are:

- Elevated triglyceride level: $\geq 1.7 \mathrm{mmol} / \mathrm{l}(150 \mathrm{mg} / \mathrm{dl})$ - decreased HDL-cholesterol: < $1.03 \mathrm{mmol} / \mathrm{l}$ (40 $\mathrm{mg} / \mathrm{dl})$ in males and $<1.29 \mathrm{mmol} / \mathrm{l}(50 \mathrm{mg} / \mathrm{dl})$ in females (or specific treatment for these abnormal lipid values).

- Elevated blood pressure (systolic $\mathrm{BP} \geq 130$ or diastolic $\mathrm{BP} \geq 85 \mathrm{mmHg}$ ) (or treatment of previously detected hypertension).

- Elevated fasting plasma glucose [FPG $\geq 5.6 \mathrm{mmol} / \mathrm{l}$ (100 mg/dl)] (or previously detected diabetes) [27].

\section{Smoking}

Participants were defined as smokers if they reported current cigarette smoking or had ceased from smoking within the last 2 years.

\section{Sample collection}

Ten milliliters $(10 \mathrm{mls})$ of blood was collected from the ante-cubital vein by venipuncture after at least a 12-h overnight fast. Four milliliters $(4 \mathrm{mls})$ was dispensed into EDTA container spun at 5000r.p.m. for $5 \mathrm{~min}$, from which $2 \mathrm{mls}$ of plasma was transferred into fluoride oxalate container for estimation of fasting plasma glucose. The remaining 6mlswas transferred into a plain container, allowed to clot at room temperature, spun at 5000r.p.m. for $5 \mathrm{~min}$; the serum was separated and cryopreserved for determination of fasting lipid profile.

\section{Biochemical analysis \\ Measurement of lipid profile parameters}

Total cholesterol (TC) was estimated using cholesterol oxidase method as described by Fredrickson et al. [28]. HDL-C was determined using Precipitation method as described by Albers et al. [29]. Triglyceride (TG) was estimated using glycerolphosphate oxidase method as described by Fossati and Prencipe [30]. The LDL-C cholesterol concentration was calculated from the total cholesterol concentration, HDL-cholesterol concentration and the triglyceride concentration using the Friedewald formula [31]:

$$
\begin{aligned}
\mathrm{LDL}-\mathrm{C}(\mathrm{mmol} / \mathrm{L})= & \text { Total cholesterol }-\mathrm{HDL}-\mathrm{C} \\
& -\mathrm{TG} / 2.2
\end{aligned}
$$

\section{Determination of fasting plasma glucose}

Fasting blood glucose was determined using glucose oxidase method. The test kit was obtained from Randox International, UK.

\section{Statistical analysis}

The data generated were analyzed using Microsoft Excel, Statistical Package for Social Science (SPSS version 20.0, California Inc.) and GraphPad prism (GraphPad Software, Inc.). Data was expressed as mean \pm standard deviation, data among groups was compared using a one-way analysis of variance (ANOVA), and post hoc analysis with Tukey's test. Correlation analysis was done using Pearson's correlation and was used to estimate inter-variable association between various selected parameters. Values of $(p<0.05)$ were considered statistically significant.

\section{Results}

Participant characteristics

Ankle brachial index (ABI), anthropometric measurements, lipid profile parameters and fasting plasma glucose (FPG) were evaluated in a total of 200 participants; 150 virologically suppressed $(\mathrm{VL}<1000$ copies $/ \mathrm{ml}$, WHO standard for Low- and middle-income countries) HIV-infected participants on antiretroviral therapy (ART) and 50 uninfected control group. Majority of the study participants were females 109 (72.7\%) while 41 (27.3\%) were males with a mean age of $43.73 \pm 8.74$ years. Based on the clinical parameters evaluated, the study participants recorded a mean systolic blood pressure (SBP) of $122 \pm 21$, diastolic blood pressure (DBP) of $81 \pm 8$, had a mean $7.73 \pm 3.52$ years duration of HIV infection, $7.47 \pm 3.42$ years duration on antiretroviral therapy, mean nadir/baseline CD4+ T-cell count of $263.18 \pm$ 229.58 cells $/ \mathrm{mm}^{3}$, mean current CD4+ T-cell count of $505.42 \pm 265.85$ cells $/ \mathrm{mm}^{3}$ and mean HIV viral RNA count of $40.95 \pm 111.61$ copies $/ \mathrm{ml}$. With regards to drug regimen, $93(61.6 \%)$ of the study participants were on the first line fixed dose regime; zidovudine/lamivudine/ nevirapine (AZT/3TC/NVP), 46 (30.5\%) were on tenofovir/lamivudine/efavirenz (TDF/3TC/EFV) while 11 (7.3\%) were on protease inhibitor; lopinavir/ritonavir (LPV/r) (Table 1).

\section{Comparison of ankle brachial index measurement and} cardiovascular risk factors in the study and control group Triglycerides and fasting plasma glucose were significantly higher in the study group compared to the control group while the control group recorded significantly higher HDL-C compared to the study group $(P<0.05)$. 
Table 1 Demographics, anthropometric parameters and clinical characteristics of virologically suppressed HIV-infected and uninfected control participants in the study

\begin{tabular}{|c|c|c|c|}
\hline Participants (n) & Study group $(n=150)$ & Control group $(n=50)$ & $P$-value \\
\hline Age (yrs.) & $43.73 \pm 8.74$ & $43.47 \pm 8.87$ & 0.714 \\
\hline \multicolumn{4}{|l|}{ Gender } \\
\hline Male (\%) & $41(27.3)$ & $23(46)$ & 0.091 \\
\hline Female (\%) & $109(72.7)$ & $27(54)$ & 0.084 \\
\hline \multicolumn{4}{|l|}{ Clinical characteristics } \\
\hline$A B \mid$ & $0.92 \pm 0.08$ & $0.94 \pm 0.05$ & 0.175 \\
\hline $\mathrm{SBP}(\mathrm{mmHg})$ & $121 \pm 21$ & $116 \pm 17$ & 0.110 \\
\hline $\mathrm{DBP}(\mathrm{mmHg})$ & $81 \pm 8$ & $76 \pm 6$ & $0.001^{*}$ \\
\hline $\mathrm{BMI}\left(\mathrm{kg} / \mathrm{m}^{2}\right)$ & $25.43 \pm 5.46$ & $25.67 \pm 4.93$ & 0.778 \\
\hline BMI $18.5-24 . \mathrm{kg} / \mathrm{m}^{2}(\%)$ & 78 (52) & $26(52)$ & 0.380 \\
\hline BMI $25-29.9$ kg/m² (\%) & $38(25.3)$ & $13(26)$ & 0.250 \\
\hline $\mathrm{BMI} \geq 30 \mathrm{~kg} / \mathrm{m}^{2}(\%)$ & $34(22.7)$ & $11(22)$ & 0.350 \\
\hline WC (cm) & $90.13 \pm 11.52$ & $90.58 \pm 11.13$ & 0.808 \\
\hline WHR & $0.88 \pm 0.048$ & $0.88 \pm 0.053$ & 0.486 \\
\hline Duration of HIV infection (yrs.) & $7.73 \pm 3.52$ & N/A & \\
\hline Duration on ART (yrs.) & $7.47 \pm 3.42$ & $\mathrm{~N} / \mathrm{A}$ & \\
\hline Nadir CD4 ${ }^{+} \mathrm{T}$ cell count (cells $/ \mathrm{mm}^{3}$ ) & $263 \pm 230$ & N/A & \\
\hline Current $\mathrm{CD} 4^{+} \mathrm{T}$ cell count (cells $/ \mathrm{mm}^{3}$ ) & $505 \pm 266$ & N/A & \\
\hline HIV viral RNA count (copies/ml) & $40.95 \pm 111.61$ & N/A & \\
\hline Undetectable viremia (<20 copies/ml) & $104(69.3)$ & & \\
\hline Detectable viremia ( $\geq 20$ copies/ml) & $46(30.7)$ & N/A & \\
\hline \multicolumn{4}{|l|}{ ART Drug regime } \\
\hline NRTI/NNRTI (AZT/3TC/NVP) (\%) & $93(62.0)$ & $\mathrm{N} / \mathrm{A}$ & \\
\hline NRTI/NNRTI (TDF/3TC/EFV) (\%) & $46(30.7)$ & N/A & \\
\hline NRTI/Protease inhibitor (TDF/3TC/LPV/r) (\%) & $11(7.3)$ & N/A & \\
\hline
\end{tabular}

Data are presented as mean \pm standard deviation or numbers (percentages). * = significant at $P<0.05$. PAD Peripheral artery disease, $A B I A n k l e$ brachial index, $S B P$ Systolic blood pressure, DBP Diastolic blood pressure, BMI Body mass index, WC Waist circumference, WHR Waist to hip ratio, CVD Cardiovascular disease, NRTI Nucleoside reverse transcriptase inhibitor; NNRTI Non-nucleoside reverse transcriptase inhibitor, AZT/3TC/NVP Zidovudine/lamivudine/nevirapine, TDF/3TC/EFV

Tenofovir/lamivudine/efavirenz, LPV/r Lopinavir/ritonavir, N/A Not applicable

Based on ankle brachial index category, the study participants recorded a higher prevalence of low ABI; 22 (14.6\%) compared to the control group $3(2 \%)$, normal ABI of 42 (27.8\%) compared to $16(10.6 \%)$ recorded by the control group and borderline ABI of 86 (57\%) compared to 31 (20.5) recorded by the control group. The study group also recorded a higher prevalence rate of metabolic syndrome; 26 (17.3\%) and diabetes mellites 6 (4\%) compared to $5(10 \%)$ for metabolic syndrome and $1(2 \%)$ for diabetes mellitus recorded by the controls. The control group however recorded higher prevalence of family history of CVD $20(13.2 \%)$ compared to the study 13 (8.6\%) (Table 2$)$.

Effect of duration on ART and drug regime class on ABI, $\mathrm{CD}^{+} \mathrm{T}$ cell count and cardiovascular risk factors in the study group

Based on duration of ART, age, ankle brachial index and current CD4+ $\mathrm{T}$ cell count significantly varied among the groups $(p<0.05)$. Study participants on ART for $\geq 10$ years recorded significantly lower ankle brachial index and higher age compared to participants on drugs for 1-5 and 6-9 years respectively (Table 3 ). Based on ART regime, waist -hip ratio, $\mathrm{ABI}$, current $\mathrm{CD} 4+\mathrm{T}$ cell count and FPG varied significantly among the three ART drug regime group. ABI and FPG were significantly higher in study participants on TDF/3TC/EFV regime compared to those on AZT/3TC/NVP. WHR was significantly higher in study participants on AZT/3TC/NVP compared to those on LPV/r (Table 4).

\section{Effect of $\mathrm{ABI}$ category on $\mathrm{CD}^{+} \mathrm{T}$ cell count and cardiovascular risk factors in the study group}

The study group stratified into 3 groups (normal $\mathrm{ABI}=$ $1-1.3$, borderline $A B I=0.9-0.99$, low $A B I=<0.9$ ) based on ankle brachial index measurement (Table 5). Total cholesterol and LDL-C significantly varied among the 3 
Table 2 Comparison of ankle brachial index and cardiovascular risk factorsbetween the study and control group using student's ttest and chi-square

\begin{tabular}{llll}
\hline Parameter & Study group $(n=150)$ & Control group $(n=50)$ & $P$-value \\
\hline ABI & $0.92 \pm 0.08$ & $0.94 \pm 0.05$ & 0.175 \\
Normal 1.0-1.3 (\%) & $42(27.8)$ & $16(10.6)$ & 0.589 \\
Borderline 0.9-0.99 (\%) & $86(57.0)$ & $31(20.5)$ & 0.562 \\
PAD $<0.90(\%)$ & $22(14.6)$ & $3(2.0)$ & $0.61 \pm 0.98$ \\
TC (mmol/L) & $4.65 \pm 0.68$ & $2.20 \pm 0.14$ & 0.453 \\
TG (mmol/L) & $2.27 \pm 0.21$ & $1.19 \pm 0.07$ & $0.001^{*}$ \\
HDL-C (mmol/L) & $1.12 \pm 0.08$ & $2.42 \pm 0.94$ & $0.001^{*}$ \\
LDL-C (mmol/L) & $2.51 \pm 0.70$ & $4.14 \pm 0.68$ & 0.236 \\
FPG (mmol/L) & $4.51 \pm 0.80$ & $20(13.2)$ & $0.001^{*}$ \\
Family history of CVD (\%) & $13(8.6)$ & $1(2.0)$ & 0.355 \\
Diabetes (\%) & $6(4.0)$ & $5(3.3)$ & 0.505 \\
Hypertension (\%) & $24(15.9)$ & $5(10.0)$ & 0.297 \\
Metabolic syndrome (\%) & $26(17.3)$ & 0.215 \\
\hline
\end{tabular}

Data are presented as mean \pm standard deviation or numbers (percentages). ${ }^{*}=$ significant at $P<0.05 . P A D$ Peripheral artery disease, $A B I$ Ankle brachial index, $S B P$ Systolic blood pressure, DBP Diastolic blood pressure, BMI Body mass index, WC Waist circumference, WHR Waist to hip ratio, CVD Cardiovascular disease, TC Total cholesterol, TG triglyceride, HDL-C High density lipoprotein cholesterol, LDL-C Low density lipoprotein cholesterol, FPG Fasting plasma glucose

Table 3 Comparison of cardiovascular risk markers and clinical characteristics based on duration on antiretroviral therapy (ART) in HIV-infected study participants using one-way ANOVA

\begin{tabular}{|c|c|c|c|c|c|c|c|}
\hline Parameter & $\begin{array}{l}\leq 5 \text { years }(n= \\
49)\end{array}$ & $\begin{array}{l}6-9 \text { years }(n= \\
50)\end{array}$ & $\begin{array}{l}\geq 10 \text { years }(n= \\
51)\end{array}$ & $\begin{array}{l}p^{-} \\
\text {value }\end{array}$ & $\begin{array}{l}\leq 5 y \text { yVVS 6-9yrs. } p- \\
\text { value }\end{array}$ & $\begin{array}{l}\leq 5 \mathrm{yrs} V S \geq 10 \mathrm{yrs} . p^{-} \\
\text {value }\end{array}$ & $\begin{array}{l}6 \text {-9yrsVS } \geq 10 \text { yrs. } p \text { - } \\
\text { value }\end{array}$ \\
\hline Age (yrs) & $41.35 \pm 9.43$ & $42.60 \pm 8.61$ & $47.14 \pm 7.13$ & $0.002^{*}$ & 0.740 & $0.002^{*}$ & $0.021^{*}$ \\
\hline$A B \mid$ & $0.93 \pm 0.07$ & $0.94 \pm 0.07$ & $0.89 \pm 0.09$ & $0.012^{*}$ & 0.715 & $0.039^{*}$ & $0.011^{*}$ \\
\hline BMI $\left(\mathrm{Kg} / \mathrm{m}^{2}\right)$ & $25.46 \pm 5.76$ & $25.37 \pm 5.61$ & $25.45 \pm 5.12$ & 0.996 & 0.996 & 1.000 & 0.997 \\
\hline WC (cm) & $88.98 \pm 11.67$ & $90.82 \pm 11.76$ & $90.55 \pm 11.29$ & 0.695 & 0.709 & 0.777 & 0.992 \\
\hline WHR & $0.87 \pm 0.06$ & $0.88 \pm 0.05$ & $0.87 \pm 0.037$ & 0.505 & 0.488 & 0.929 & 0.711 \\
\hline SBP $(\mathrm{mmHg})$ & $120 \pm 21$ & $118 \pm 18$ & $127 \pm 22$ & 0.054 & 0.833 & 0.189 & 0.053 \\
\hline DBP (mmHg) & $82 \pm 8$ & $80 \pm 7.27$ & $82 \pm 8$ & 0.128 & 0.176 & 0.189 & 0.191 \\
\hline $\begin{array}{l}\text { Nadir CD4 } \\
\text { cell (cells/ } \\
\mathrm{mm}^{3} \text { ) }\end{array}$ & $210 \pm 224$ & $272 \pm 210$ & $304 \pm 247$ & 0.122 & 0.382 & 0.106 & 0.754 \\
\hline $\begin{array}{l}\text { Current CD4 } \\
\mathrm{T} \\
\text { cell (cells/ } \\
\mathrm{mm}^{3} \text { ) }\end{array}$ & $352 \pm 220$ & $560 \pm 265$ & $596 \pm 247$ & $0.001^{*}$ & $0.001^{*}$ & $0.001^{*}$ & 0.745 \\
\hline FPG (mmol/L) & $4.40 \pm 0.52$ & $4.61 \pm 1.16$ & $4.53 \pm 0.54$ & 0.413 & 0.383 & 0.709 & 0.850 \\
\hline TC $(\mathrm{mmol} / \mathrm{L})$ & $4.43 \pm 1.12$ & $4.84 \pm 1.02$ & $4.57 \pm 0.74$ & 0.112 & 0.101 & 0.774 & 0.343 \\
\hline TG (mmol/L) & $2.17 \pm 0.15$ & $2.22 \pm 0.14$ & $2.19 \pm 0.14$ & 0.141 & 0.127 & 0.397 & 0.387 \\
\hline $\begin{array}{l}\mathrm{HDL}-\mathrm{C}(\mathrm{mmol} / \\
\mathrm{L})\end{array}$ & $1.19 \pm 0.07$ & $1.20 \pm 0.06$ & $1.19 \pm 0.06$ & 0.582 & 0.884 & 0.841 & 0.552 \\
\hline $\begin{array}{l}\text { LDL-C (mmol/ } \\
\text { L) }\end{array}$ & $2.25 \pm 1.07$ & $2.62 \pm 0.99$ & $2.38 \pm 0.72$ & 0.141 & 0.125 & 0.764 & 0.409 \\
\hline
\end{tabular}

Data are presented as mean \pm standard deviation. ${ }^{*}=$ significant at $P<0.05$. PAD Peripheral artery disease, $A B I$ Ankle brachial index, SBP Systolic blood pressure, DBP Diastolic blood pressure, BMI Body mass index, WC Waist circumference, WHR Waist to hip ratio, CVD Cardiovascular disease, TC Total cholesterol, TG Triglyceride, HDL-C High density lipoprotein cholesterol, LDL-C Low density lipoprotein cholesterol, FPG Fasting plasma glucose 
Table 4 Statistical comparison of traditional cardiovascular risk markers and clinical characteristics based on ART drug regime in thestudy participants using one-way ANOVA

\begin{tabular}{|c|c|c|c|c|c|c|c|}
\hline Parameter & $\begin{array}{l}\text { AZT/3TC/NVP } \\
(n=93)\end{array}$ & $\begin{array}{l}\text { TDF/3TC/EFV } \\
(n=46)\end{array}$ & $\begin{array}{l}\mathrm{TDF} / 3 \mathrm{TC} / \mathrm{LPV} / \mathrm{r} \\
(n=11)\end{array}$ & $\begin{array}{l}p- \\
\text { value }\end{array}$ & $\begin{array}{l}\text { AZT/3TC/NVP Vs TDF/ } \\
\text { 3TC/EFV p-value }\end{array}$ & $\begin{array}{l}\text { AZT/3TC/NVP Vs TDF/ } \\
\text { 3TC/LPV/r p-value }\end{array}$ & $\begin{array}{l}\text { TDF/3TC/EFV Vs TDF/ } \\
3 \mathrm{TC/LPV/r} \text { p-value }\end{array}$ \\
\hline BMI $\left(\mathrm{Kg} / \mathrm{m}^{2}\right)$ & $25.71 \pm 5.54$ & $25.19 \pm 5.18$ & $24.04 \pm 6.15$ & 0.599 & 0.865 & 0.606 & 0.803 \\
\hline WC (cm) & $90.30 \pm 11.17$ & $90.09 \pm 11.49$ & $88.82 \pm 15.29$ & 0.922 & 0.994 & 0.915 & 0.943 \\
\hline WHR & $0.88 \pm 0.04$ & $0.87 \pm 0.05$ & $0.85 \pm 0.06$ & $0.041^{*}$ & 0.530 & $0.039^{*}$ & 0.216 \\
\hline SBP (mmHg) & $123 \pm 20$ & $122 \pm 25$ & $112 \pm 11$ & 0.270 & 0.994 & 0.2 .43 & 0.306 \\
\hline $\mathrm{DBP}(\mathrm{mmHg})$ & $82 \pm 8$ & $81 \pm 7$ & $76 \pm 5$ & 0.073 & 0.823 & 0.059 & 0.152 \\
\hline$A B I$ & $0.91 \pm 0.09$ & $0.94 \pm 0.06$ & $0.92 \pm 0.09$ & $0.041^{*}$ & $0.039^{*}$ & 0.873 & 0.671 \\
\hline $\begin{array}{l}\text { Nadir CD4+ T cells } \\
\text { (cells } / \mathrm{mm}^{3} \text { ) }\end{array}$ & $295 \pm 250$ & $211 \pm 177$ & $216 \pm 211$ & 0.100 & 0.106 & 0.998 & 0.998 \\
\hline $\begin{array}{l}\text { Current CD4+ T cells } \\
\left.\text { (cells } / \mathrm{mm}^{3}\right)\end{array}$ & $549 \pm 272$ & $443 \pm 245$ & $393 \pm 237$ & $0.029^{*}$ & $0.041^{*}$ & 0.149 & 0.834 \\
\hline FPG (mmol/L) & $4.40 \pm 0.56$ & $4.79 \pm 1.13$ & $4.32 \pm 0.54$ & $0.019^{*}$ & $0.020^{*}$ & 0.938 & 0.179 \\
\hline TC (mmol/L) & $4.68 \pm 1.04$ & $4.46 \pm 0.90$ & $4.66 \pm 0.60$ & 0.432 & 0.404 & 0.997 & 0.810 \\
\hline TG (mmol/L) & $2.20 \pm 0.15$ & $2.19 \pm 0.14$ & $2.23 \pm 0.09$ & 0.671 & 0.919 & 0.754 & 0.647 \\
\hline $\mathrm{HDL}-\mathrm{C}(\mathrm{mmol} / \mathrm{L})$ & $1.20 \pm 0.07$ & $1.19 \pm 0.06$ & $1.18 \pm 0.08$ & 0.538 & 0.611 & 0.730 & 0.976 \\
\hline LDL-C (mmol/L) & $2.49 \pm 1.01$ & $2.28 \pm 0.09$ & $2.46 \pm 0.54$ & 0.460 & 0.432 & 0.997 & 0.823 \\
\hline
\end{tabular}

Data are presented as mean \pm standard deviation. ${ }^{*}=$ significant at $P<0.05$. $P A D$ Peripheral artery disease, $A B I$ Ankle brachial index, SBP Systolic blood pressure, $D B P$ Diastolic blood pressure, BMI Body mass index, WC Waist circumference, WHR Waist to hip ratio, CVD Cardiovascular disease, TC Total cholesterol, TG Triglyceride, HDL-C High density lipoprotein cholesterol, LDL-C Low density lipoprotein cholesterol, FPG Fasting plasma glucose

group $(P<0.05)$. Total cholesterol and LDL-C were significantly higher in participants with low ABI compared to those with borderline ABI. LDL-C was significantly higher in study participants with low ABI compared to those who demonstrated normal ABI (Table 5).

\section{Association of low ankle brachial index with cardiovascular risk factors and clinical characteristics in the study group}

Low ankle brachial index showed significant association with total cholesterol (OR 1.784, $P=0.011$ ) and low-

Table 5 Statistical analysis of anthropometric parameters, clinical characteristics, lipid profile, fasting plasma glucose based on ankle brachial index (ABI) measurement in the study participants

\begin{tabular}{|c|c|c|c|c|c|c|c|}
\hline Parameter & $\begin{array}{l}\text { Normal ABI }(1-1.3) \\
(n=42)\end{array}$ & $\begin{array}{l}\text { Borderline ABI }(0.9- \\
0.99)(n=86)\end{array}$ & $\begin{array}{l}\operatorname{PAD}(>0.9) \\
(n=22)\end{array}$ & $\begin{array}{l}P- \\
\text { value }\end{array}$ & $\begin{array}{l}\text { Normal ABI VSborderline } \\
\text { ABI } P \text {-value }\end{array}$ & $\begin{array}{l}\text { Borderline } \\
\text { ABI VS } \\
\text { PAD P-value }\end{array}$ & $\begin{array}{l}\text { Normal ABI VS PAD } \\
\text { P-value }\end{array}$ \\
\hline Age (yrs) & $44.67 \pm 7.85$ & $43.43 \pm 9.50$ & $43.14 \pm 7.32$ & 0.713 & 0.735 & 0.989 & 0.786 \\
\hline $\mathrm{BMI}\left(\mathrm{Kg} / \mathrm{m}^{2}\right)$ & $25.43 \pm 6.05$ & $25.08 \pm 5.31$ & $26.77 \pm 4.86$ & 0.435 & 0.941 & 0.401 & 0.618 \\
\hline WC $(\mathrm{cm})$ & $89.55 \pm 12.10$ & $89.28 \pm 11.38$ & $94.55 \pm 10.39$ & 0.149 & 0.991 & 0.135 & 0.991 \\
\hline WHR & $0.87 \pm 0.05$ & $0.87 \pm 0.05$ & $0.89 \pm 0.04$ & 0.468 & 1.000 & 0.455 & 0.532 \\
\hline Nadir CD4 & $281 \pm 230$ & $258 \pm 242$ & $250 \pm 176$ & 0.833 & 0.853 & 0.990 & 0.853 \\
\hline Current CD4 & $495 \pm 248$ & $507 \pm 277$ & $518 \pm 267$ & 0.946 & 0.970 & 0.985 & 0.945 \\
\hline $\begin{array}{l}\text { Duration on } \\
\text { ART (yrs) }\end{array}$ & $6.88 \pm 3.14$ & $7.55 \pm 3.45$ & $8.32 \pm 3.75$ & 0.479 & 0.555 & 0.611 & 0.248 \\
\hline $\mathrm{FPG}(\mathrm{mmHg})$ & $4.57 \pm 1.20$ & $4.49 \pm 0.56$ & $4.50 \pm 0.62$ & 0.268 & 0.848 & 0.998 & 0.938 \\
\hline $\mathrm{TC}(\mathrm{mmol} / \mathrm{L})$ & $4.58 \pm 1.02$ & $4.50 \pm 0.99$ & $5.15 \pm 0.62$ & $0.019 *$ & 0.893 & $0.014^{*}$ & 0.066 \\
\hline $\mathrm{TG}(\mathrm{mmol} / \mathrm{L})$ & $2.21 \pm 0.16$ & $2.19 \pm 0.15$ & $2.23 \pm 0.08$ & 0.397 & 0.766 & 0.390 & 0.768 \\
\hline $\begin{array}{l}\mathrm{HDL}-\mathrm{C} \text { (mmol/ } \\
\mathrm{L})\end{array}$ & $1.20 \pm 0.06$ & $1.19 \pm 0.07$ & $1.19 \pm 0.06$ & 0.942 & 0.948 & 0.942 & 1.000 \\
\hline $\begin{array}{l}\text { LDL-C (mmol/ } \\
\mathrm{L})\end{array}$ & $2.38 \pm 1.00$ & $2.31 \pm 0.95$ & $2.94 \pm 0.64$ & $0.017^{*}$ & 0.907 & $0.013^{*}$ & $0.038^{*}$ \\
\hline
\end{tabular}

Data are presented as mean \pm standard deviation. ${ }^{*}=$ significant at $P<0.05$. ABI Ankle brachial index, SBP Systolic blood pressure, DBP Diastolic blood pressure, $B M I$ Body mass index, WC Waist circumference, WHR Waist to hip ratio, CVD Cardiovascular disease, TC Total cholesterol, TG Triglyceride, HDL-C high density lipoprotein cholesterol, LDL-C Low density lipoprotein cholesterol, FPG Fasting plasma glucose 
density lipoprotein cholesterol (OR 1.824, $P=0.010$ ). TG (OR 6.440, $P=0.250$ ), CD4 T cell $<200$ copies $/ \mathrm{ml}$ (OR 2.635, $P=0.250$ ), WHR (OR 928.39, $P=0.250$ ), hypertension (OR 2.115, $P=0.335$ ), detectable viraemia $\geq 20$ copies/ml (OR 1.375, $P=0.511$ ) demonstrated high odd ratio for predicting low ABI in the study group (Table 6).

\section{Correlation analysis between duration on ART and ankle brachial index, triglyceride and viral load in the study group}

Figure 1 shows a significant positive correlation $(r=0.202$, $p=0.013$ ) between triglyceride and viral load. Figure 2 shows a significant negative correlation $(r=-0.163, P=$ 0.041 ) between duration on ART and ankle brachial index (ABI). This represents an inverse relationship between $\mathrm{ABI}$ and duration on ART.

\section{Discussion}

Effectively treated HIV-infected patients still have an elevated risk for cardiovascular morbidity and mortality, which is believed to be associated not only to traditional risk factors, but also to dyslipidemia initiated by HIV and/or

Table 6 Results of logistic regression analysis: association of risk factors with low ankle-brachial index value in the study group

\begin{tabular}{|c|c|c|c|}
\hline Parameter & OR & $95 \% \mathrm{Cl}$ & $P$-value \\
\hline$\overline{\mathrm{BMI}}$ & 1.052 & $0.971-1.138$ & 0.214 \\
\hline Age & 0.993 & $0.942-1.046$ & 0.703 \\
\hline WC & 1.040 & $1.000-1.082$ & 0.052 \\
\hline WHR & 1.126 & $0.409-3.107$ & 0.818 \\
\hline CD4+ T cell $\geq 500$ cells $/ \mathrm{mm}^{3}$ & 1.000 & $0.999-1.002$ & 0.801 \\
\hline CD4+ T cell $<200$ cells $/ \mathrm{mm}^{3}$ & 2.635 & $0.327-21.05$ & 0.364 \\
\hline Duration of HIV & 1.060 & $0.929-1.210$ & 0.385 \\
\hline Duration on ART & 1.094 & $0.952-1.258$ & 0.203 \\
\hline FPG & 0.978 & $0.546-1.753$ & 0.941 \\
\hline TC & 1.784 & $1.142-2.787$ & $0.011^{*}$ \\
\hline TG & 6.440 & $0.269-154.26$ & 0.250 \\
\hline HDL-C & 0.215 & $0.000-208.20$ & 0.215 \\
\hline LDL-C & 1.824 & $1.155-2.880$ & $0.010^{*}$ \\
\hline Hypertension & 2.115 & $0.460-9.716$ & 0.335 \\
\hline Family history of CVD & 0.956 & $0.197-4.642$ & 0.956 \\
\hline MetS based on IDF & 1.051 & $0.324-3.408$ & 0.935 \\
\hline Protease inhibitor (LPV/r) & 0.678 & $0.134-3.43$ & 0.638 \\
\hline Hypertension & 2.115 & $0.461-9.716$ & 0.335 \\
\hline Diabetes mellitus & 0.328 & $0.056-1.910$ & 0.215 \\
\hline Viral load $\geq 20$ copies/ml & 1.375 & $0.532-3.55$ & 0.511 \\
\hline
\end{tabular}

$*$ significant at $P<0.05$. Cl Confidence interval, $O R$ Odd ratio, TC Total cholesterol, TG Triglyceride, BMI Body mass index, FPG Fasting plasma glucose, WC Waist circumference, WHR Waist hip ratio, HDL High-density lipoprotein $L D L$ Low-density lipoprotein, LPV/r Lopinavir/ritonavir antiretroviral treatment [24]. In the general population, carotid intima-media thickness (IMT) evaluated using ultrasonography has been demonstrated to correlate with coronary atherosclerosis [32], and directly associated in older patients with an elevated risk of myocardial infarction and stroke in those without a history of cardiovascular disease [33]. Carotid IMT measurement, notwithstanding, is costly and not easily accessible, instead a noninvasive technique used is the ankle-brachial index (ABI), a simple inexpensive diagnostic test that is a strong indicator of systemic atherosclerosis and peripheral vascular disease and a powerful predictor of mortality from cardiovascular events in the general population [34, 35].

This study evaluated the prevalence of peripheral artery disease (PAD) using ankle brachial index measurement $(\mathrm{ABI}<0.9)$ and its associated risk factors in virologically suppressed HIV-infected participants attending their routine out-patient clinic reviews in University of Ilorin Teaching Hospital and Sabo Oke Medical center Ilorin, Kwara state, Nigeria. The study population was relatively young with a mean age of $43.73 \pm 8.74$ years and mainly composed of female participants $(72.7 \%)$. The prevalence of metabolic syndrome, as recorded in study population group using the IDF criteria, is $17.3 \%$ compared to $10 \%$ recorded in the control group. These findings are consistent with studies of Katherine et al. [36], who reported a prevalence of $14-18 \%$ among HIV-infected patients, a study conducted Pirjo et al. [37], also revealed higher prevalence of metabolic syndrome in HIV-infected individuals compared to the uninfected controls with a prevalence rate of $10-14 \%$ among HIV-infected individuals with normal glucose tolerance. Treatment with ART can induce severe metabolic complications including lipodystrophy, dyslipidaemia, and insulin resistance and may contribute to the higher prevalence rate observed in the study group [38].

PAD has been shown to correlate with future incident cardiovascular events particularly ischemic strokes and myocardial infarction [39], and can be evaluated using ABI, a low ABI of $<0.9$ is suggestive of PAD. A study conducted by Gutierrez et al. [40], showed a relationship between low $\mathrm{ABI}$ and elevated carotid intima media thickness, suggesting that in HIV-infected patients, low ABI may be a significant predictor of subclinical atherosclerosis. In this present study, Ankle brachial index was significantly lower in the study group compared to the HIV-negative control group. The study group recorded higher prevalence rate of low ABI; $14.6 \%$ compared to $2 \%$ prevalence rate observed in the control group. This is consistent with reports by Periard et al. [41], who reported a high prevalence rate $(20.7 \%)$ of PAD among HIV patients on ART in a Swiss cohort. Findings from this study are also in line with the works of Beckman et al. [42], who reported a $19 \%$ increased risk of PAD among HIV-infected veterans. 


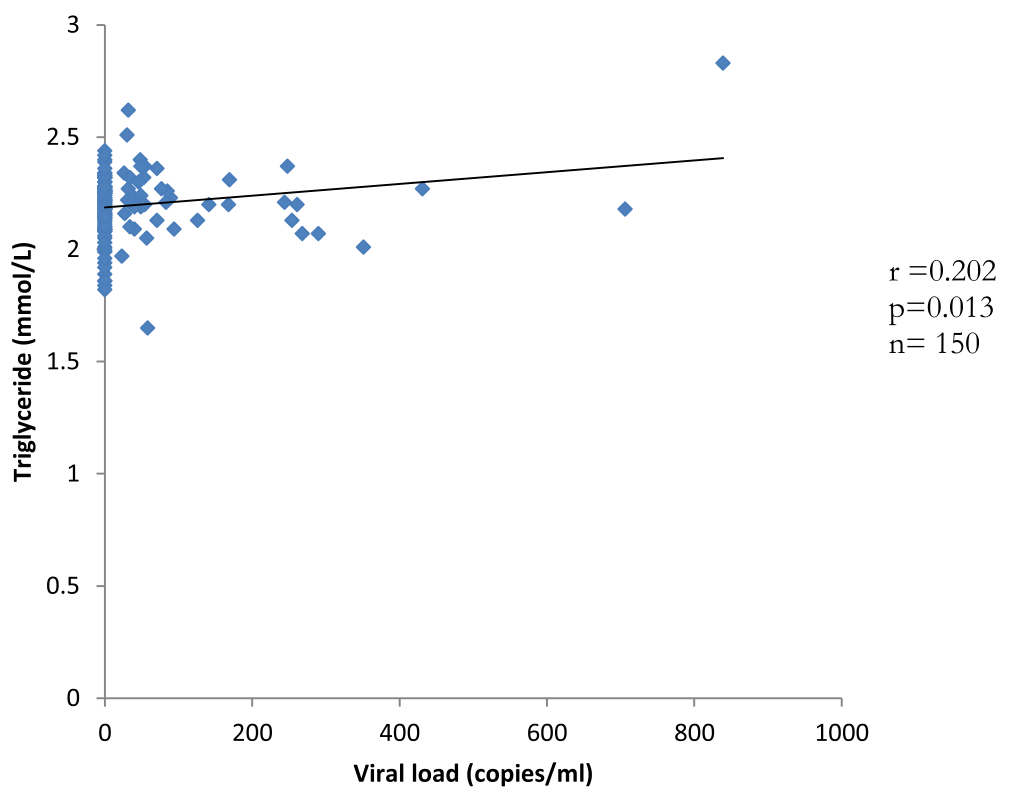

Fig. 1 Correlation plot of triglyceride against viral load in HIV-infected study group. The preliminary analysis of the data showed that a significantly positive correlation was observed between triglyceride and viral RNA count

Different mechanisms may be linked to the premature development of PAD in HIV-infected population. People living with HIV tend to have elevated prevalence of traditional cardiovascular risk factors compared to the uninfected controls, this was observed in this study as HIV-infected study group demonstrated higher prevalence rate of hypertension $15.9 \%$, metabolic syndrome $17.3 \%$, diabetes mellitus $4 \%$ compared to the prevalence rate recorded by the control group; hypertension $3.3 \%$, metabolic syndrome 10 and $2 \%$ for diabetes mellitus.
These risk factors have been linked to future events of atherosclerosis and coronary heart disease. In a study carried out by Guiterrez et al. [40], results showed that traditional CVD risk factors such as hypertension, dyslipidaemia and current smoking status were linked with increased intima media thickness (IMT) within HIV-infected study group.

Another possible mechanism may be linked to combination antiretroviral therapy-related dyslipidaemia, lipodystrophy, and impaired glucose linked to the development

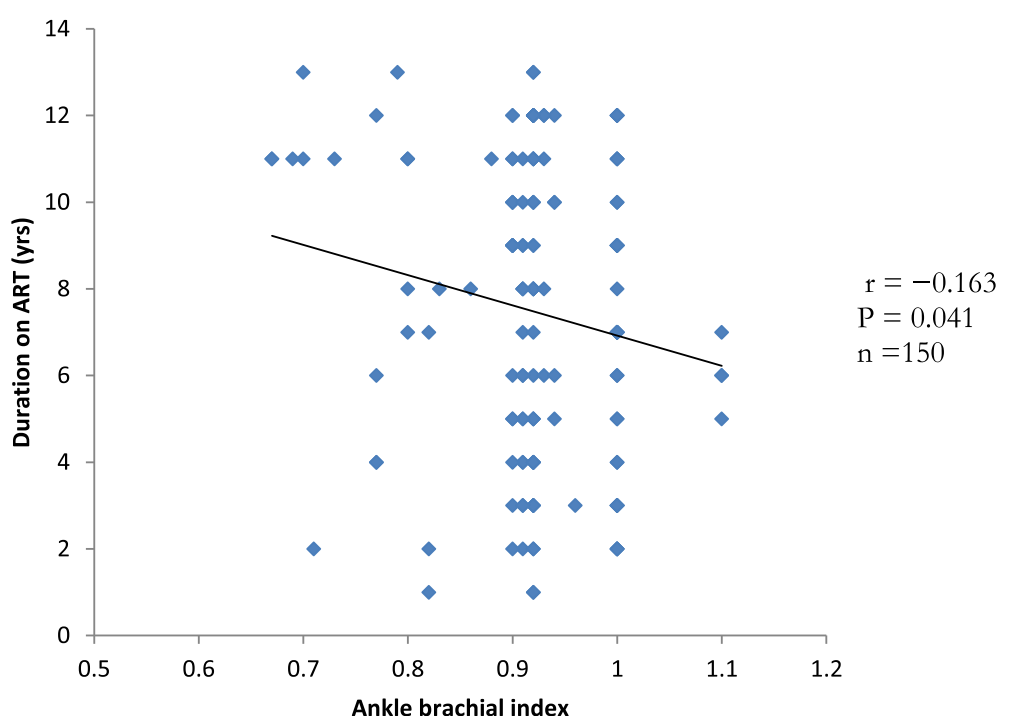

Fig. 2 Correlation plot of Duration on ART against ankle brachial index (ABI) in HIV-infected study group. The preliminary analysis of the data showed that a significantly negative correlation was observed between duration of ART (years) and ankle brachial index in the study group 
of premature atherosclerosis, as previously demonstrated by Maggi et al. [43], and Depairon et al. [44], especially in those on protease inhibitors (PIs). In contrast to these reports, the use of PI did not show a strong associated with low $\mathrm{ABI}$ in the present study group. This discrepancy may be due to the mechanisms by which PIs are atherogenic. PIs are associated with usually reversible dyslipidemia, and its long-term may lead to development of atherosclerosis, whereas a short duration of PI exposure may temporarily modify lipid levels without substantial development of atherosclerosis [44]. A negative correlation was however observed between ABI and the duration on ART in this study, this indicates that there is an association between ART and PAD, which needs further clarification. Larger studies with larger number of patients will be necessary to confirm the role of ART and to investigate possible associations of other antiviral agents with PAD.

Another mechanism may result from the direct damage caused by HIV on the arterial wall, leading to inflammatory lesions, as recently demonstrated by Maggi et al. [45]. This is represented by the aneurysmal dilation of large arteries seen in HIV-infected children. In HIV-infected children aged 2-9 years who developed HIV through vertical transmission, [46]. The aortic root size was related with left ventricular dilation, decreased CD4+ T-cell count and elevated viral load. Furthermore, cases of 13 HIV-infected children with cerebral artery aneurysms were reported by Dubrovsky et al., [47]. Reports from this study revealed that CD $4<200$ copies $/ \mathrm{mm}^{3}$ was associated with a two-fold elevation in the risk of PAD while detectable viraemia was associated with a one-fold increase in the risk of PAD. Such an association has previously been reported by Mercie' et al. [48], but the underlying mechanism is not clear. These clinical outcomes observed in this study have been supported by other studies that used surrogate CVD markers. In the Women's Interagency HIV Study (WIHS) and Multicenter AIDS Cohort Study (MACS), a current CD4+ T-cell count $<200$ cells/ $\mathrm{mm}^{3}$ was linked to carotid plaque (defined as a carotid IMT $1.5 \mathrm{~mm}$ ) [49]. In most of the antiretroviral-treated individuals observed in earlier San Francisco-based research, a nadir CD4+ T-cell count $<200$ cells/mm3 was also independently related to carotid IMT [50]. The association of PAD with reduced CD4+ T-cell count may also be linked to patient age, as older age is a risk factor of reduced robust increase in CD4+ T-cell counts after initiation of antiretroviral therapy.

Findings from this study revealed that total cholesterol; LDL-C and TG were significantly higher in the HIV- infected study group compared to the uninfected control group. This is in line with the study of Nsonwu et al. [51], who also reported similar findings. Dyslipidaemia in HIV-infected individuals has been attributed to elevated apolipoprotein levels, increased hepatic synthesis of TG and VLDL-C, decreased clearance of TG, acute-phase proteins, and to the effects of viral infection itself. The levels of total cholesterol (TC) and high-density lipoproteincholesterol (HDL-C) has been shown to be rather decreased in the early stages of HIV infection [52]. The progressive decrease in $\mathrm{CD} 4+\mathrm{T}$ cell lymphocyte counts resulting from destruction by HIV has been however demonstrated to induce a decreased clearance of LDL-C particles, a decrease in HDL-C and an increase in the TG levels which may be positively linked to the level of viraemia [53]. ART has also been shown to affect the metabolism of triglyceriderich lipoproteins [54].

In addition to the above findings, HIV-infected study participants with altered (low) ABI values recorded significantly higher mean values of TC and LDL-C compared to study participants with normal and borderline $\mathrm{ABI}$ values and together with TG predicted low ABI in the study group. These findings may be linked to the fact that LDL-C accumulates in the intima of the artery and initiates the vascular inflammatory process [55]. Oxidized LDL-C and turbulent blood flow occurring at vascular branch points can stimulate adhesion molecules on the endothelial surface of the artery [56]; the stimulation of these adhesion molecules causes activated macrophages and $\mathrm{T}$ cells to infiltrate the arterial walls [57]. Monocytes differentiate into macrophages, which take up oxidized LDL-C, forming foam cells, which is critical in the final formation of atheroma's and development to cardiovascular diseases.

Among HIV-infected participants in this study, the levels of HDL-C were significantly lower compared to un-infected control group. These findings are consistent with the report of Nsonwu et al. [51], who reported that lower levels of HDL among HIV-infected individuals on ART compared to uninfected controls. Lower HDL-C levels may result from HIV infection and/or thrombotic activity, a mediator of endothelial injury and thrombogenesis, or both. The activity of CETP (cholesterol ester transfer protein), which transfers cholesterol esters from HDL-C to apolipoprotein-B-containing proteins [58], is elevated in HIV infection, and its activity inversely correlates with serum HDL levels [59]. This may assist in explaining why HDL-C levels are reduced in HIV infection. Although the reason for elevated CETP activity is still uncertain; CETP functions more efficiently when there is a increased TG level [60], and may aid in explaining the increased activity in HIV-infected patients.

\section{Conclusion}

Detectable viral load count and long term treatment with antiretroviral therapy are associated with dyslipidemia, with elevated risk of peripheral artery disease. Prevalence of PAD in virologically-suppressed HIV-infected participants did not differ from the HIV-negative controls in the population studied. 


\section{Limitations of the study}

Our present study is limited by its small sample size and larger studies are required to better characterize risk factors for PAD and its prognosis in this population. There was also no possibility of comparing our results with others in the Nigerian general population as we did not find them in the literature. Accordingly, in the discussion of our results, we compared them with results of the most important worldwide research concerning the prevalence of PAD in the general population. Lastly, this was a single center study in a tertiary hospital; therefore our findings cannot be generalized.

\section{Additional file}

Additional file 1: Questionnairè. (DOCX 15 kb)

\section{Abbreviations}

ABI: Ankle brachial index; AIDS: Acquired Immune Deficiency Syndrome; ART: Antiretroviral therapy; cIMT: Carotid Intima media thickness; CVD: Cardiovascular disease; ELISA: Enzyme-Linked Immunosorbent Assay; HDL-C: high-density lipoprotein cholesterol; HIV: Human Immunodeficiency Virus; LDL-C: Low-density lipoprotein cholesterol; MACS: Multicenter AIDS Cohort Study; NCDs: Non Communicable Diseases; PAD: Peripheral Artery Disease; PLHIV: People Living with Human Immunodeficiency Virus; sICAM1: Soluble intercellular adhesion molecule-1; TG: Triglyceride; WIHS: Women's Inter-agency HIV Study

\section{Acknowledgments}

We thank our study participants for spending their time with us during data collection period. We also thank families, friends and HIV/AIDS organization for their assistance during the study period. We gratefully would not fail to thank specially the management and staff of HIV clinic in University of Ilorin Teaching Hospital (UITH) and Sabo Oke medical center, Ilorin, Kwara state, Nigeria.

\section{Authors' contributions}

CEA conceived the research and obtained the data. CEA and PUA participated equally in the design of the study and performed the analysis. IKU, CAN and CNO contributed analysis tools and copy-edited the manuscript. All authors prepared, read and approved the manuscript.

\section{Funding}

This research did not receive any specific grant from any funding agency or organization.

\section{Availability of data and materials}

The datasets used and/or analyzed during the current study are available from the corresponding author on reasonable request.

\section{Ethics approval and consent to participate}

The research ethical approval was obtained from the Kwara State Ministry of Health Ethical Committee (MOH/KS/EU/777/252). Enrollment into the study was voluntary. Informed consent was written and only consenting participants were recruited for the study.

\section{Consent for publication}

Not applicable.

\section{Competing interests}

The authors declare that they have no competing interests.

\section{Author details}

'Prime Health Response Initiative (PHRI)-sub-recipients of Global Fund HIV Impact Project, Ilorin, Kwara State, Nigeria. ${ }^{2}$ Department of Medical Laboratory Science, Faculty of Health Science and Technology, College of
Medicine, University of Nigeria Enugu Campus, Enugu, Nigeria. ${ }^{3}$ Department of Medical Laboratory Science, Faculty of Allied Medical Science, University of Calabar, Calabar, Cross River State, Nigeria.

Received: 7 February 2019 Accepted: 14 August 2019

Published online: 20 August 2019

\section{References}

1. Luz PM, Bruyand M, Ribeiro S. IPEC/FIOCRUZ Cohort and the Aquitaine ANRS C03 Study Group. AIDS and non-AIDS severe morbidity associated with hospitalizations among HIV-infected patients in two regions with universal access to care and antiretroviral therapy, France and Brazil, 20002008: hospital-based cohort studies. BMC Infect Dis. 2014;14(5):278.

2. Lima VD, Hogg RS, Harrigan PR. Continued improvement in survival among HIV-infected individuals with newer forms of highly active antiretroviral therapy. AIDS. 2007;21(6):685-92.

3. Palella FJ Jr, Baker RK, Moorman AC, HIV Outpatient Study Investigators. Mortality in the highly active antiretroviral therapy era: changing causes of death and disease in the HIV outpatient study. J Acquir Immune Defic Syndr. 2006;43(1):27-34

4. Mocroft A, Reiss P, Gasiorowski J. Euro SIDA Study Group, serious fatal and nonfatal non-AIDS-defining illnesses in Europe. J Acquir Immune Defic Syndr. 2010;55(2):262-70

5. French AL, Gawel SH, Hershow R. Trends in mortality and causes of death among women with HIV in the United States: a 10-year study. J Acquir Immune Defic Syndr. 2009:51(4):399-406.

6. Berry SA, Fleishman JA, Moore RD, Gebo KA, HIV Research Network. Trends in reasons for hospitalization in a multisite United States cohort of persons living with HIV, 2001-2008. J Acquir Immune Defic Syndr. 2012;59(4):368-75.

7. Triant VA, Lee H, Hadigan C, Grinspoon SK. Increased acute myocardial infarction rates and cardiovascular risk factors among patients with human immunodeficiency virus disease. J Clin Endocrinol Metab. 2007;92(7):2506-12.

8. Saves M, Chene G, Ducimetiere P, Leport C, Le Moal G, et al. Risk factors for coronary heart disease in patients treated for human immunodeficiency virus compared with the general population. Clin Infect Dis. 2003;37(2):292-8.

9. Muronya W, Sanga E, Talama G, Kumwenda J, Joep J. Cardiovascular risk factors in adult Malawians on long-term antiretroviral therapy. Am J Obstet Gynecol. 2011;105(11):644-9.

10. Bloomfield G, Alenezi F, Barasa F, Lumsden R, Mayosi B, Velazquez E. Human Immunodeficiency Virus and Heart Failure in Low- and Middle-Income Countries. JACC Heart Fail. 2015;3(8):579-90.

11. Groenewald P, Nannan N, Bourne D, Laubscher R, Bradshaw D. Identifying death from AIDS in South Africa. AIDS. 2005;19:193-201.

12. Friis-Moller $\mathrm{N}$, Thiebaut $\mathrm{R}$, Reiss $\mathrm{P}$, Weber $\mathrm{R}$, Monforte $\mathrm{AD}$, De Wit $\mathrm{S}$, et al Predicting the risk of cardiovascular disease in HIV-infected patients: the data collection on adverse effects of anti-HIV drugs study. Eur J Cardiovasc Prev Rehabil. 2010;17:491-501.

13. Deeks SG. HIV infection, inflammation, immunosenescence, and aging. Annu Rev Med. 2011;62:141-55.

14. Ingle SM, May MT, Gill MJ, Mugavero MJ, Lewden C, Abgrall S, et al. Impact of risk factors for specific causes of death in the first and subsequent years of antiretroviral therapy among HIV-infected patients. Clin Infect Dis. 2014;59(4):287-97.

15. Ogden CL, Carroll MD, Curtin LR, McDowell MA, Tabak CJ, Flegal KM. Prevalence of overweight and obesity in the united states, 1999-2004 JAMA. 2006;295:1549-55.

16. Reinecke $H$, Unrath M, Freisinger E, Bunzemeier $H$, Meyborg M, Luders F, et al. Peripheral arterial disease and critical limb ischaemia: still poor outcomes and lack of guideline adherence. Eur Heart J. 2015;36(15):932-8.

17. Hooi JD, Kester AD, Stoffers HE, Rinkens PELM, Knottnerus JA, Van Ree JW, et al. Asymptomatic peripheral arterial occlusive disease predicted cardiovascular morbidity and mortality in a 7-year follow- up study. J Clin Epidemiol. 2004;57(3):294-300.

18. Norgren L, Hiatt WR, Dormandy JA, Nehler MR, Harris KA. TASC II Working Group: Inter-Society Consensus for the Management of Peripheral Artery Disease (TASC II). J Vasc Surg. 2007;45(Suppl S):5-67.

19. Aboyans V, Criqui MH, McClelland RL, Allison MA, McDermott MM, Goff DC, et al. Intrinsic contribution of gender and ethnicity to normal ankle-brachial index values: The Multi-Ethnic Study of Atherosclerosis (MESA). J Vasc Surg. 2007:45:319-27.

20. National Population Commission. 'Enumerators manual', March. Third Report of the Expert Panel on Detection, Evaluation and Treatment of High Blood 
Cholesterol in Adults (Adult Treatment Panel III). The guidelines 2006. National Heart Lung and Blood Institute. Available: http://www.nhlbi.nih. gov/guidelines/cholesterol/index.html. Accessed 2.01.2019.

21. Friis-Moller N, Weber R, Reiss P, Thiebaut R, Kirk O, d'Aminio Monforte A, et al, Cardiovascular disease risk factors in HIV patients-association with antiretroviral therapy. Results from the D:A:D study. AIDS. 2003;17(8):1179-93.

22. Kwiatkowska W, Knysz B, Arczynska K, Drelichowski J, Czarnecki M, Gasiorowski J, et al. Peripheral arterial disease and ankle-brachial index abnormalites in young and middle-aged HIV-positive patients in Lower Silesia, Poland. PLoS One. 2014;12:01-18.

23. Olalla J, Salas D, Del Arco A, De la Torre J, Prada JL, Machin HS, et al. Ankle brachial index and HIV: the role of antiretrovirals. Br HIV Assoc HIV Med. 2009:10:1-5.

24. Schienkiewitz A, Mensink GBM, Scheidt-Nave C. Comorbidity of overweight and obesity in a nationally representative sample of German adults aged 18-79 years. BMC Public Health. 2012;12:658.

25. Agu C, Usoro C, Inaku K, Nsonwu A, Offor S. Cross-sectional analysis of cardiovascular risk markers among obese males and females in Southern Nigeria. Scholars J Appl Med Sci. 2016;4(5A):1471-8.

26. Alberti K, Zimmet $P$, Shaw J. Metabolic syndrome: a new world-wide definition. A Consensus Statement from the International Diabetes Federation. Diabet Med. 2006;23:469-80.

27. The IDF consensus worldwide definition of the metabolic syndrome International Diabetes Federation. Available: http://www.idf.org/webdata/ docs/MetS_def_FINAL.pdf. Accessed 2.01.2019

28. Fredrickson DS, Levy RL, Lees RS. Fat transportin lipoproteins-An integrated approach to mechanisms and disorders. N Engl J Med. 1967;276:273-81.

29. Albers JJ, Warnick GR, Chenng MC. Quantitation of high density lipoproteins. Lipids. 1978;13(12):926-32.

30. Fossati P, Prencipe L. Serum triglycerides determined colorimetrically with an Enzyme that produces hydrogen peroxide. Clin Chem. 1982;28:2077-80

31. Friedewald WT, Levy RI, Fredrickson DS. Estimation of the Concentration of Low-Density Lipoprotein Cholesterol in Plasma, Without Use of the Preparative Ultracentrifuge. Clin Chem. 1972;18(6):499-502.

32. Kablak-Ziembicka A, Tracz W, Przewlocki T, Pieniazek P, Sokolowski A, Konieczynska M. Association of increased carotidintima-media thickness with the extent of coronary artery disease. Heart. 2004;90:1286-90.

33. O'Leary DH, Polak JF, Kronmal RA, Manolio TA, Burke GL, Wolfson SK Jr. Carotid-artery intima and media thicknessas a risk factor for myocardial infarction and stroke in olderadults. Cardiovascular Health Study Collaborative ResearchGroup. N Engl J Med. 1999;340:14-22.

34. Doobay AV, Anand SS. Sensitivity and specificity of the ankle brachial index to predict future cardiovascular outcomes: a systematic review. ArteriosclerThrombVasc Biol. 2005;25:1463-9.

35. McDermott MM, Liu K, Criqui MH, Ruth K, Goff D, Saad MF, et al. Anklebrachial index and subclinical cardiac and carotiddisease: the multiethnic study of atherosclerosis. Am J Epidemiol. 2005;162:33-41.

36. Katherine S, Handan W, Matthew L. Prevalence of metabolic syndrome in HIV-infected patients receiving highly active antiretroviral therapy using International Diabetes Foundation and Adult Treatment Panel III criteria. Diabetes Care. 2007:30:113-9.

37. Pirjo IL, Johan GE, Jaan L. Prevalence of metabolic syndrome and its complication. Diabetes care. 2004;27(9):2135-40.

38. Grundy SM, Brewer HB, Cleeman Jl, Smith SC Jr, Lenfant C, et al. Definition of metabolic syndrome: Report of the National Heart, Lung, and Blood Institute/American Heart Association conference on scientific issues related to definition. Circulation. 2004;109:433-8.

39. Poredos $P$, Jug B. The prevalence of peripheral arterial disease in high risk subjects and coronary or cerebrovascular patients. Angiology. 2007;58:309-15.

40. Gutierrez F, Enrique B, Sergio P, Ildefonso MM. Relationship between anklebrachial index and carotid intima-media thickness in HIV-infected patients. AIDS. 2008;22:1369-76.

41. Periard D, Cavassini M, Taffe P, Chevalley M, Senn L, Chapuis T, et al. High prevalence of peripheral arterial disease in HIV-infected persons. Clin Infect Dis. 2008;46:761-7.

42. Beckman J, Duncan M, Alcorn C, So-Armah K, Butt A, Goetz M. Association of Human Immunodeficiency Virus Infection and Risk of Peripheral Artery Disease. Circulation. 2018;138:255-65.

43. Maggi P, Serio G, Epifani G, Fiorentino G, Saracino A, Fico C, et al. Premature lesions of the carotid vessels in HIV-1-infected patients treated with protease inhibitors. AIDS. 2000;14(16):123-8.
44. Depairon M, Chessex S, Sudre P, Rodondi N, Doser N, Chave JP, et al. Premature atherosclerosis in HIV-infected individuals - focus on protease inhibitor therapy. AIDS. 2001;15(3):329-34.

45. Maggi P, Perilli F, Lillo A, Carito V, Epifani G, Bellacosa C, et al. An ultrasound-based comparative study on carotid plaques in HIVpositive patients vs. atherosclerotic and arteritis patients: atherosclerotic or inflammatory lesions? Coron Artery Dis. 2007;18:23-9.

46. Lai WW, Colan SD, Easley KA, Lipshultz SE, Starc TJ, Bricker JT, et al. Dilation of the aortic root in children infected with human immunodeficiency virus type 1: The Prospective P2C2 HIV Multicenter Study. Am Heart J. 2001;141:661-70.

47. Dubrovsky T, Curless R, Scott G, Chaneles M, Post MJ, Altman N, et al. Cerebral aneurysmal arteriopathy in childhood AIDS. Neurology. 1998;51:560-5.

48. Mercie P, Thiebaut R, Aurillac-Lavignole V, et al. Carotid intima-media thickness is slightly increased over time in HIV-1-infected patients. HIV Med. 2005:6:380-6387.

49. Kaplan RC, Kingsley LA, Gange SJ, Benning L, Jacobson LP, Lazar J, et al. Low CD4+ T-cell count as a major atherosclerosis risk factor in HIV-infected women and men. AIDS. 2008;22:1615-24.

50. Hsue PY, Lo JC, Franklin A, Bolger AF, Martin JN, Deeks SG, et al. Progression of atherosclerosis as assessed by carotid intima-media thickness in patients with HIV infection. Circulation. 2004;109:1603-8.

51. Nsonwu-Anyanwu A, Egbe E, Agu C, Offor J, Usoro C, Essien I, et al. Nutritional Indices and Cardiovascular Risk Factors in HIV Infection in Southern Nigeria. J Immunol Clin Microbiol. 2017;2(2):34-42.

52. Cunha J, Maselli LMF, Stern ACB, Spada C, Bydlowski SP. Impact of antiretroviral therapy on lipid metabolism of human immunodeficiency virus-infected patients: Old and new drugs. World J Virol. 2015;4:56-77.

53. Cerrato E, Calcagno A, D'Ascenzo F, Biondi-Zoccai G. Cardiovascular disease in HIV patients: from bench to bedside and backwards. Open Heart. 2015;2:e000174.

54. Mulligan K, Grunfeld C, Tai WW, Algren H. Hyperlipidemia and insulin resistance are induced by protease inhibitors independent of changes in body composition in patients with HIV infection. J Acquir Immune DeficSyndr. 2000;23:35-43.

55. Matsuura E, Hughes GR, Khamashta MA. Oxidation of LDL and its clinical implication. Autoimmun Rev. 2008:7:558-66.

56. Navab M, Ananthramaiah GM, Reddy ST, Van Lenten BJ, Ansell BJ, Fonarow GC, et al. The oxidation hypothesis of atherogenesis: the role of oxidized phospholipids and HDL. J Lipid Res. 2004;45:993-1007.

57. Kádár A, Glasz T. Development of atherosclerosis and plaque biology. Cardiovasc Surg. 2001;9:109-21.

58. Lewis GF, Rader DJ. New insights into the regulation of HDL metabolism and reverse cholesterol transport. Circ Res. 2005;96:1221-32.

59. Guerin M, Le Goff W, Lassel TS, Van Tol A, Steiner G, Chapman MJ. Atherogenic role of elevated CE transfer from HDL to VLDL (1) and dense LDL in type 2 diabetes: impact of the degree of triglyceridemia. Arterioscler ThrombVasc Biol. 2001;21:282-8.

60. Laura KP, Dennis LS, Max CW, Frank LT. Mechanism of inhibition defines CETP activity: a mathematical model for CETP in vitro. J Lipid Res. 2009; 50(11):2222-34.

\section{Publisher's Note}

Springer Nature remains neutral with regard to jurisdictional claims in published maps and institutional affiliations.

Ready to submit your research? Choose BMC and benefit from:

- fast, convenient online submission

- thorough peer review by experienced researchers in your field

- rapid publication on acceptance

- support for research data, including large and complex data types

- gold Open Access which fosters wider collaboration and increased citations

- maximum visibility for your research: over $100 \mathrm{M}$ website views per year

At $\mathrm{BMC}$, research is always in progress.

Learn more biomedcentral.com/submission 